\title{
New Evidence for the Mechanism of Action of a Type-2 Diabetes Drug Using a Magnetic Bead-Based Automated Biosensing Platform
}

Uddin, Rokon; Nur-E-Habiba; Rena, Graham; Hwu, En Te; Boisen, Anja

Published in:

ACS Sensors

Link to article, DOI:

10.1021/acssensors.7b00384

Publication date:

2017

Document Version

Publisher's PDF, also known as Version of record

Link back to DTU Orbit

Citation (APA):

Uddin, R., Nur-E-Habiba, Rena, G., Hwu, E. T., \& Boisen, A. (2017). New Evidence for the Mechanism of Action of a Type-2 Diabetes Drug Using a Magnetic Bead-Based Automated Biosensing Platform. ACS Sensors, 2(9), 1329-1336. https://doi.org/10.1021/acssensors.7b00384

\section{General rights}

Copyright and moral rights for the publications made accessible in the public portal are retained by the authors and/or other copyright owners and it is a condition of accessing publications that users recognise and abide by the legal requirements associated with these rights.

- Users may download and print one copy of any publication from the public portal for the purpose of private study or research.

- You may not further distribute the material or use it for any profit-making activity or commercial gain

- You may freely distribute the URL identifying the publication in the public portal 


\title{
New Evidence for the Mechanism of Action of a Type-2 Diabetes Drug Using a Magnetic Bead-Based Automated Biosensing Platform
}

\author{
Rokon Uddin, *†॰ Nur-E-Habiba, ${ }^{\ddagger}$ Graham Rena, ${ }^{\S}$ En-Te Hwu, ${ }^{\prime \prime}$ and Anja Boisen ${ }^{\dagger}$ \\ ${ }^{\dagger}$ Department of Micro- and Nanotechnology, Technical University of Denmark, DTU Nanotech, Building 345 East, DK-2800 \\ Kongens Lyngby, Denmark \\ ${ }^{\ddagger}$ Department of Chemistry and Bioengineering, Tampere University of Technology, 33720, Tampere, Finland \\ ${ }^{\S}$ Molecular and Clinical Medicine, School of Medicine, University of Dundee, Dundee DD1 9SY, United Kingdom \\ "Institute of Physics, Academia Sinica, Nankang, 11529 Taiwan
}

Supporting Information

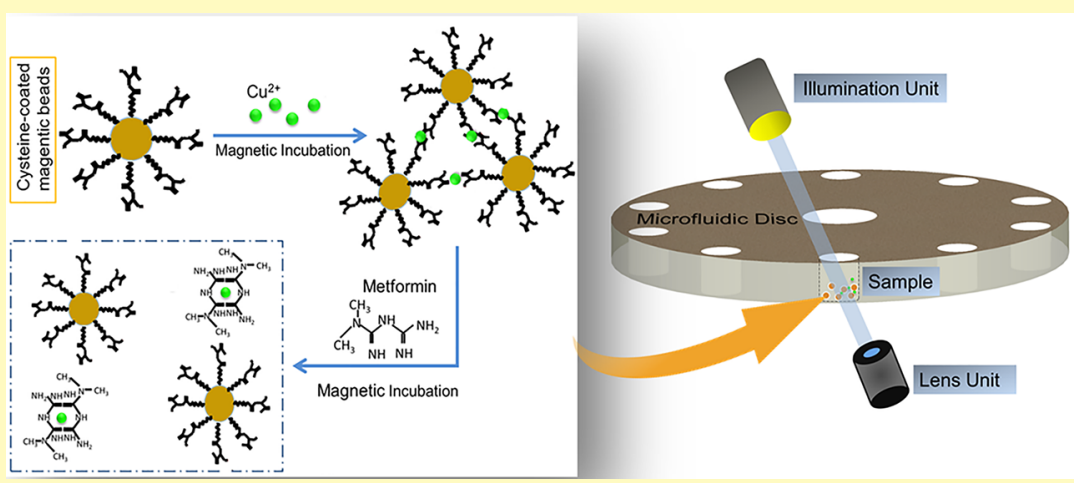

ABSTRACT: The mechanism of action (MOA) of the first line type-2 diabetes drug metformin remains unclear despite its widespread usage. However, recent evidence suggests that the mitochondrial copper $(\mathrm{Cu})$-binding action of metformin may contribute toward the drug's MOA. Here, we present a novel biosensing platform for investigating the MOA of metformin using a magnetic microbead-based agglutination assay which has allowed us to demonstrate for the first time the interaction between $\mathrm{Cu}$ and metformin at clinically relevant low micromolar concentrations of the drug, thus suggesting a potential pathway of metformin's blood-glucose lowering action. In this assay, cysteine-functionalized magnetic beadswere agglutinated in the presence of $\mathrm{Cu}$ due to cysteine's $\mathrm{Cu}$-chelation property. Addition of clinically relevant doses of metformin resulted in disaggregation of $\mathrm{Cu}$-bridged bead-clusters, whereas the effect of adding a closely related but blood-glucose neutral drug propanediimidamide (PDI) showed completely different responses to the clusters. The entire assay was integrated in an automated microfluidics platform with an advanced optical imaging unit by which we investigated these aggregationdisaggregation phenomena in a reliable, automated, and user-friendly fashion with total assay time of 17 min requiring a sample (metformin/PDI) volume of $30 \mu \mathrm{L}$. The marked difference of Cu-binding action between the blood-glucose lowering drug metformin and its inactive analogue PDI thus suggests that metformin's distinctive Cu-binding properties may be required for its effect on glucose homeostasis. The novel automated platform demonstrating this novel investigation thus holds the potential to be utilized for investigating significant and sensitive molecular interactions via magnetic bead-based agglutination assay.

KEYWORDS: biosensor, type-2 diabetes, metformin, magnetic beads, agglutination assay, optical imaging, molecular interactions

415 million people have diabetes worldwide, and by 2040 , the number will rise to 642 million according to the latest estimates of the International Diabetes Federation (IDF); at least $90 \%$ of these patients have type- 2 diabetes. Metformin was first used in humans as a type-2 diabetes (T2D) drug in the late $1950 \mathrm{~s}^{1,2}$ and is the first-line oral treatment for T2D. As it was discovered before the modern day target-based drug discovery era, its mechanism of action at the molecular level was not established before its clinical use and still remains unclear today. ${ }^{1,3,4} \mathrm{~A}$ detailed understanding of its molecular mechanism might lead to the development of next generation target-based drug for treating T2D. Regarding the molecular mechanism of metformin, studies have shown that treatment of liver cells with metformin leads to reduced mitochondrial respiration ${ }^{5-8}$ and concomitant reduction of hepatic energy through depletion of intracellular ATP content. ${ }^{9}$ Thus, due to lower hepatic energy, a lower amount of glucose is produced by hepatic cells. However, the pathway through which metformin reduces the

Received: June 6, 2017

Accepted: August 4, 2017

Published: August 4, 2017 
mitochondrial respiration is not established yet and is an area of vigorous research.

This drug has been proven to interact with different metals ${ }^{10-14}$ and the most stable of these interactions is with the metal copper $(\mathrm{Cu}) .^{15,16}$ Recently the relationship of $\mathrm{Cu}$ binding to the clinical action of metformin has been under investigation. $^{16-18}$ The studies found that metformin binds with mitochondrial $\mathrm{Cu}$ under conditions where it inhibits mitochondrial respiration. Both $\mathrm{Cu}^{2+}$ and $\mathrm{Cu}^{1+}$ ions are important ions in electron transport chain enzymes during ATP production, ${ }^{19-21}$ and inhibition of these enzymes will reduce the content of intracellular ATP, resulting in less glucose production due to lower hepatic energy. ${ }^{7}$ Up to now, however, the cell studies with metformin, including those described above, have depended on high (1-2 $\mathrm{mM}$ or more) concentrations of drug, and therefore it is unclear if they are related to lowering of the glucose level in blood (antihyperglycemia), which are mediated by low micromolar plasma concentrations of the drug in plasma. Consequently, in the current study we have developed tools to measure metformin's metal-binding properties at therapeutically relevant concentrations.

Magnetic bead-based assays are being utilized for different applications like detection of small molecules, ${ }^{22,23}$ pathogens, ${ }^{24}$ and proteins, ${ }^{25,26}$ as well as PCR amplification ${ }^{27}$ and drug delivery. $^{28,29}$ Detection of biomarkers or molecules by agglutination-based sensing using magnetic beads is being used for its readout simplicity. ${ }^{30-34}$ Hence, in our previous preliminary study, ${ }^{17}$ the concept of our well-studied magnetic bead-based agglutination assay ${ }^{22,31,32,34,35}$ had been utilized for investigating the effects of metformin on $\mathrm{Cu}$ ions as a step toward understanding the mechanism of metformin. As $\mathrm{Cu}$ ions hardly remain as free ions in the cells ${ }^{36-38}$ and approximately $35 \%$ of them remain bound to cysteine residues, ${ }^{39,40}$ we prepared $\mathrm{Cu}-\mathrm{L}$-cysteine compound/aggregates using nanosized magnetic beads to mimic at least $35 \%$ of $\mathrm{Cu}$-ligands of the cell, as these magnetic nanobead (MNB) aggregates will model protein-bound copper ions particularly well. Addition of different concentrations of metformin caused dissolution of the aggregates indicating breakage of the Cu-L-cysteine bonds. The detection of the aggregation-disaggregation phenomena was performed by a Blu-ray-based optomagnetic system ${ }^{32,34}$ where the signal peaks were sensitive to the size and concentration of the aggregates. Thus, the previous agglutination assay demonstrated that metformin interacts with cysteinebound $\mathrm{Cu}$-ligand. A significant drawback, however, is that it was insufficiently sensitive to study this property at physiological concentrations (micromolar) of the drug. In addition, it did not demonstrate the evidence of relationship between the $\mathrm{Cu}$ binding action and its glucose lowering property.

In this paper, we present an integrated and automated biosensing platform with a substantial improvement in sensitivity compared to our previous work while using microsized magnetic beads (hereafter represented by "MB"). These improvements have allowed us to quantify, for the first time, the effects of micromolar concentrations of metformin on copper-bridged cysteine $\mathrm{MB}$ aggregates. To investigate how well metformin's Cu-binding action corresponds with its glucose lowering property, we have compared the $\mathrm{Cu}$ metformin interactions with an inactive analogue of the drug propanediimidamide (PDI). Although structurally similar to metformin, PDI does not show antihyperglycemic behavior in cells $^{16,17}$ nor does it exhibit anti-inflammatory effects noted recently for metformin. ${ }^{41}$ The completely different behavior of PDI compared to that of metformin suggests that metformin's Cu-binding action may be required for its glucose-lowering effect. The overall study has been performed with control experiments to demonstrate the specificity of metformin's interaction with $\mathrm{Cu}$-bridged MBs. Furthermore, the experimental setup has been improved compared to our previous study ${ }^{18}$ by integrating an automated optical imaging unit (oCelloScope, Philips Biocell) for automated operation of the entire study, allowing rapid optimization of the $\mathrm{Cu}-\mathrm{MB}$ clusters. The usage of microscale beads aided visualization and the characterization of the clusters using the imaging unit assisted in the process of the optimization of the assay protocol. The developed sensing platform has the potential to be an out-of-lab setting for studying molecular interactions through MB-based agglutination assay.

\section{EXPERIMENTAL SECTION}

Materials and Chemicals. The MBs (Dynabeads MyOne Streptavidin T1, $10 \mathrm{mg} / \mathrm{mL}$ ) with a surface-coating of streptavidin used in this study were bought from Life Technologies (Thermo Scientific). EZ-Link NHS-LC-LC Biotin was ordered from VWR (Thermo Scientific, Product no. 21343) and L-cysteine was purchased from Merck KGaA (Germany). $50 \mathrm{mM}$ PBS (pH 7.4) and $50 \mathrm{mM}$ MES ( $\mathrm{pH}$ 6.0) buffer were prepared using Milli-Q water for washing and sample preparation. The drug vehicle MES buffer was prepared by dissolving 0.488 g MES hydrate (Sigma-Aldrich, product no. M8250; 2-( $\mathrm{N}$-morpholino) ethanesulfonic acid) in $45 \mathrm{~mL}$ Milli-Q water while the $\mathrm{pH}$ was adjusted with $1 \mathrm{M} \mathrm{NaOH} . \mathrm{Cu}\left(\mathrm{NO}_{3}\right)_{2}$ purchased from Sigma-Aldrich was prepared as $0.5 \mathrm{mM}$ solution in MES buffer for performing experiments. Metformin (1,1-dimethylbiguanide hydrochloride) and PDI (propanediimidamide dihydrochloride) purchased from Sigma-Aldrich were prepared at different concentration in MES buffer.

Sample Preparation. A solution of $10 \mathrm{mM}$ biotin and a solution of 0.6 M L-cysteine were prepared using PBS buffer ( $\mathrm{pH}$ 7.4). Next, the solutions of biotin and L-cysteine were mixed with a volume ratio of $1: 8.5$ involving $30 \mathrm{~min}$ incubation at room temperature (RT) for biotinylating the L-cysteine. Next, $10 \mathrm{mg} / \mathrm{mL}$ microsized MBs (bead diameter $1 \mu \mathrm{m}$ ) coated with streptavidin was washed three times with PBS buffer ( $\mathrm{pH}$ 7.4). After that, the MB solution, biotinylated Lcysteine solution and PBS buffer were mixed with a volume ratio of 1:3.5:5.5 followed by incubation of $1 \mathrm{~h}$ with gentle shaking on a shaker at RT. Finally, the incubated solution was washed three times with 50 $\mathrm{mM}$ MES buffer and resuspended at a bead concentration of $1 \mathrm{mg} /$ $\mathrm{mL}$.

Biosensing Platform. In our previous study, ${ }^{18}$ we used a microfluidic disc-based optomagnetic unit for demonstrating the effects of different concentrations of metformin on the clusters formed through $\mathrm{Cu}$ and magnetic nanobeads (MNB). In the current study, we have integrated an optical imaging unit ${ }^{34,42}$ onto the disc-based optomagnetic unit in order to integrate visualization and quantification of the effects of metformin on the microsized Cu-MB clusters (Figure 1). The setup, in addition, facilitates the integration and automation of fluidics operation along with automated detection in a user-friendly fashion.

The current setup (Figure 1) consists of a removable microfluidic disc, a pair of permanent magnets mounted onto the platform for performing magnetic incubation (MI), and an optomagnetic unit along with an optical imaging unit for visualization and quantification of nano/microparticles. The disc is attached to a closed-loop motor (Maxxon Motor, mod. 273756, Switzerland) and controlled using a LabVIEW (National Instruments) based program which facilitates automation of the assay and reproducible positioning of the sample. The optical imaging unit can be moved along both the horizontal and vertical axes using the instrumental software (Uniexplorer 6.0) which facilitates automated scanning at the precise positions of the sample/ disc ensuring reproducibility for multiple measurements. The 


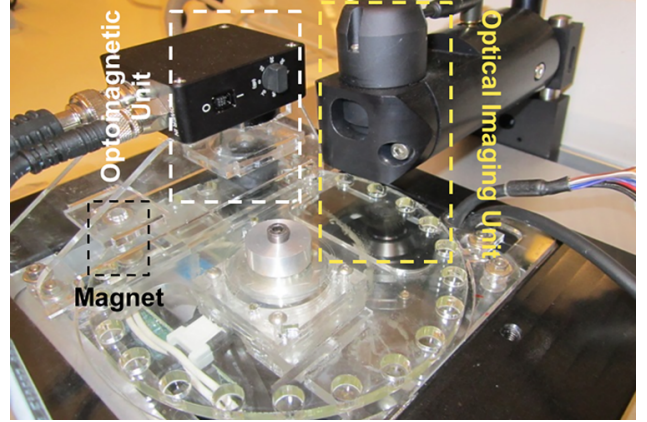

Figure 1. Integrated platform for studying molecular interactions. Integrated experimental setup consisting of a Blu-ray based optomagnetic unit, an automated optical imaging unit, and a pair of permanent magnets on a centrifugal microfluidics platform. Details of the detection units can be found in ref 43 .

automated scanning creates multiple stacks of images using optical sectioning principle of confocal microscopy resulting in capture of all the $\mathrm{MBs} / \mathrm{MB}$ clusters in focus within the scanning window. Using the instrumental image processing algorithm, the software calculates the size, i.e., the projected area of each $\mathrm{MBs} / \mathrm{MB}$ aggregates, and thus gives the mean $\mathrm{MB}$ aggregate size in a sample. Further details of the scanning principle can be found in refs 34 and 42 .

Experimental Procedure. The assay platform in this study was a microfluidic disc made from two layers of poly(methyl methacrylate) (PMMA) and bonded by a layer of pressure sensitive adhesive (PSA). The disc (thickness $4.7 \mathrm{~mm}$ ) contains 24 wells each of which is $4 \mathrm{~mm}$ in depth and $7 \mathrm{~mm}$ in diameter (Figure 2a). Thus, 24 individual experiments can be performed in a single disc, the fabrication of time of which is $<10 \mathrm{~min}$. Further information on the disc fabrication is presented in the Supporting Information Section S1.

The experimental setup has three defined positions: sample-loading position, magnetic incubation (MI) position, and scanning position (Figure 3). First, the microfluidic disc was mounted onto the experimental setup and the well of the disc in which the $\mathrm{Cu}-\mathrm{MB}$ sample was to be loaded was placed at the sample-loading position. Then, a mixed solution of $\mathrm{Cu}$ and streptavidin-coated MBs functionalized with biotinylated L-cysteine (volume ratio 11:1) was loaded into the particular well of the disc (Figure 2b). After sample loading, the disc rotated following a rotation routine set by the LabVIEW program until the sample-filled well reached the position between the two permanent magnets (MI position) for performing MI for 6 min with a magnetic field of $60 \mathrm{mT}$. The MI protocol consisted of constant incubation (10 s) under the magnet for enhancing agglutination, followed by clockwise and anticlockwise shaking of the disc with a speed of $30 \mathrm{rpm}$ for breaking unspecific binding as well as facilitating interaction between $\mathrm{MBs}$ and $\mathrm{Cu}$. Due to the $\mathrm{Cu}$-chelation

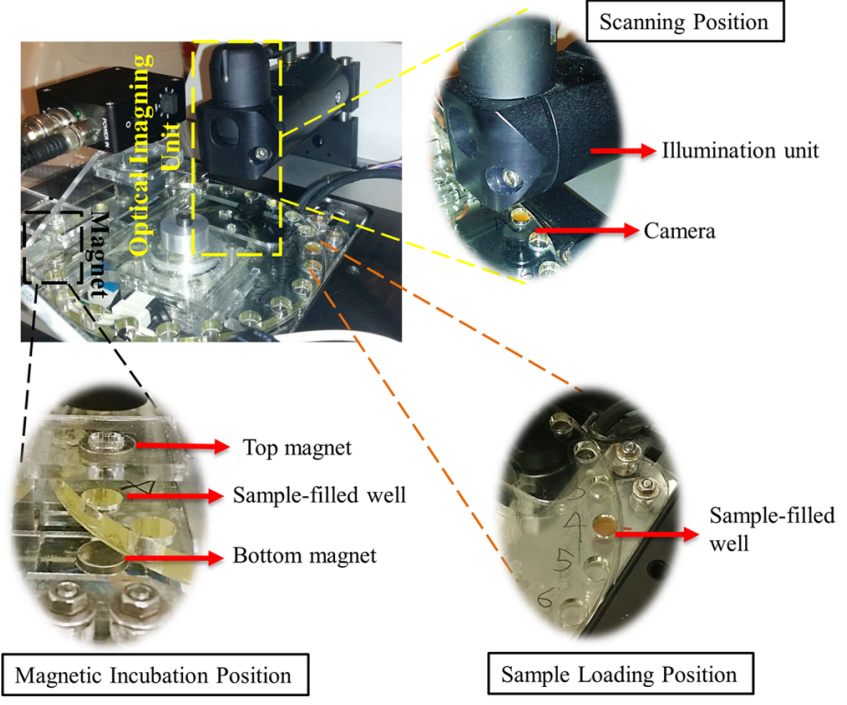

Figure 3. Detailed illustration of different significant positons of the experimental setup.

property of L-cysteine, the MBs functionalized with L-cysteine bridge with $\mathrm{Cu}$ through $\mathrm{Cu}$-L-cysteine bond and, thus, causes the formation of $\mathrm{Cu}-\mathrm{MB}$ clusters (Figure $2 \mathrm{~b}$ ). The complete MI protocol was run by the rotation routine made in the LabVIEW program facilitating reproducibility of the incubation. After the completion of MI, the disc rotated following the rotation routine for the sample-filled well to reach the precise position (scanning position) between the light source and camera of the optical imaging unit in order to perform scanning of the clusters. The solution was scanned and the captured images were analyzed by Uniexplorer 6.0 to quantify the $\mathrm{Cu}-\mathrm{MB}$ clusters. After scanning, the sample-filled well was again positioned to the sampleloading position using the software in order to add $30 \mu \mathrm{L}$ of different concentration of metformin/PDI into the $\mathrm{Cu}-\mathrm{MB}$ clusters (volume ratio $1: 2)$. After adding the sample, the same rotation routine was initiated to perform MI followed by scanning to quantify the clusters. The purpose of the second MI was to further ensure the agglutination as well as facilitating interaction between $\mathrm{Cu}-\mathrm{MB}$ clusters and metformin/PDI while breaking the nonspecific bindings. The total assay time including incubation and scanning was approximately 17 $\min$.

\section{RESULTS AND DISCUSSION}

Interaction of Metformin and PDI with Copper. Our previous study using MNBs and the optomagnetic unit of the experimental setup ${ }^{18}$ showed that $\mathrm{Cu}-\mathrm{MNB}$ clusters formed
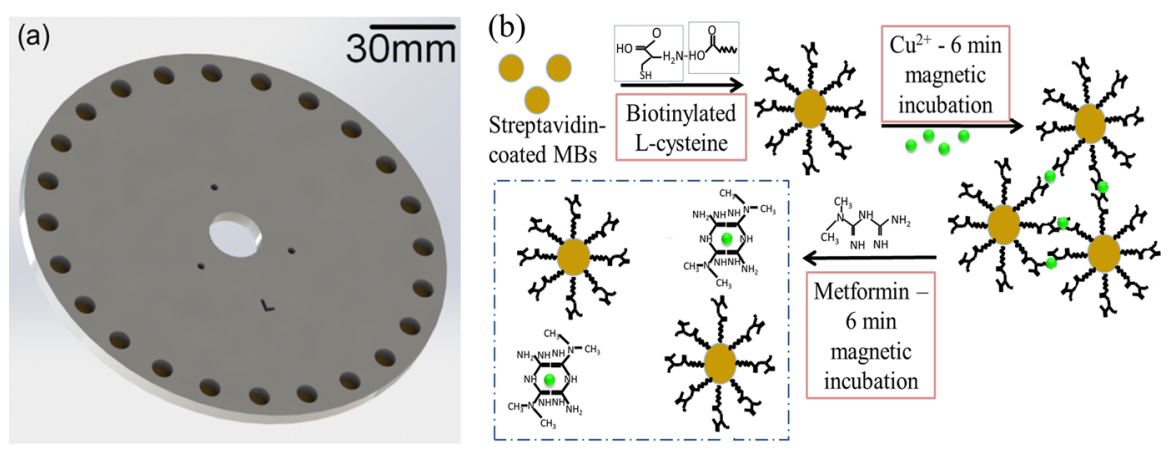

Figure 2. Schematic of disc and assay design. (a) Schematic of the microfluidic disc consisting of 24 wells where the agglutination assay is performed. (b) Schematic of the agglutination assay. The MBs functionalized with biotinylated L-cysteine forms clusters through L-cysteine-Cu bond after the addition of $\mathrm{Cu}^{2+}$ followed by magnetic incubation, which are again disaggregated after adding metformin followed by the second magnetic incubation. Further information on the assay scheme is presented in the Supporting Information Section S2. 
(a)
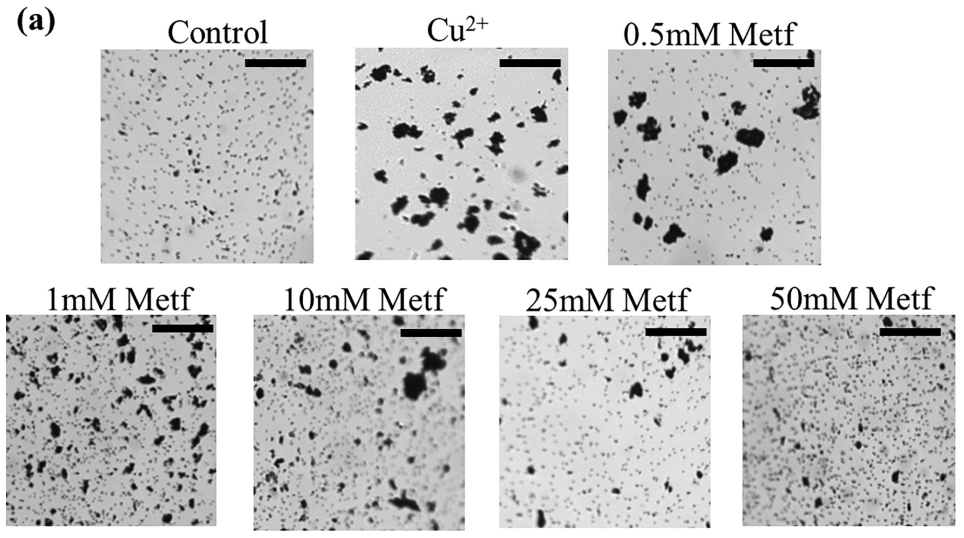

(b)

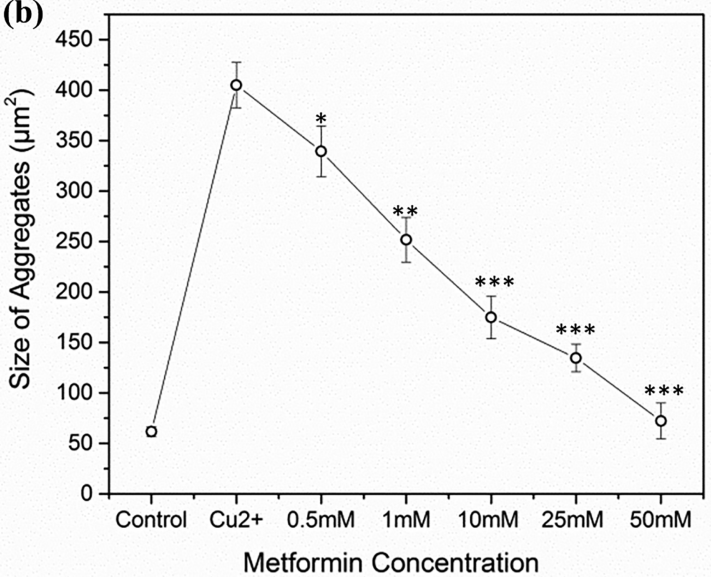

Figure 4. Effect of adding metformin (Metf) at different concentrations into the Cu-MB clusters. (a) Images captured by the optical imaging unit of different $\mathrm{MB}$ samples. (b) Corresponding mean area of $\mathrm{MB}$ aggregates (calculated by Uniexplorer 6.0) vs different MB samples. Error bars indicate the standard deviation obtained from triplicate measurements. Scale bar: $50 \mu \mathrm{m}$. $* P<0.05, * * P<0.01, * * * P<0.001$ by one way ANOVA test compared to $\mathrm{Cu}^{2+}$ sample.
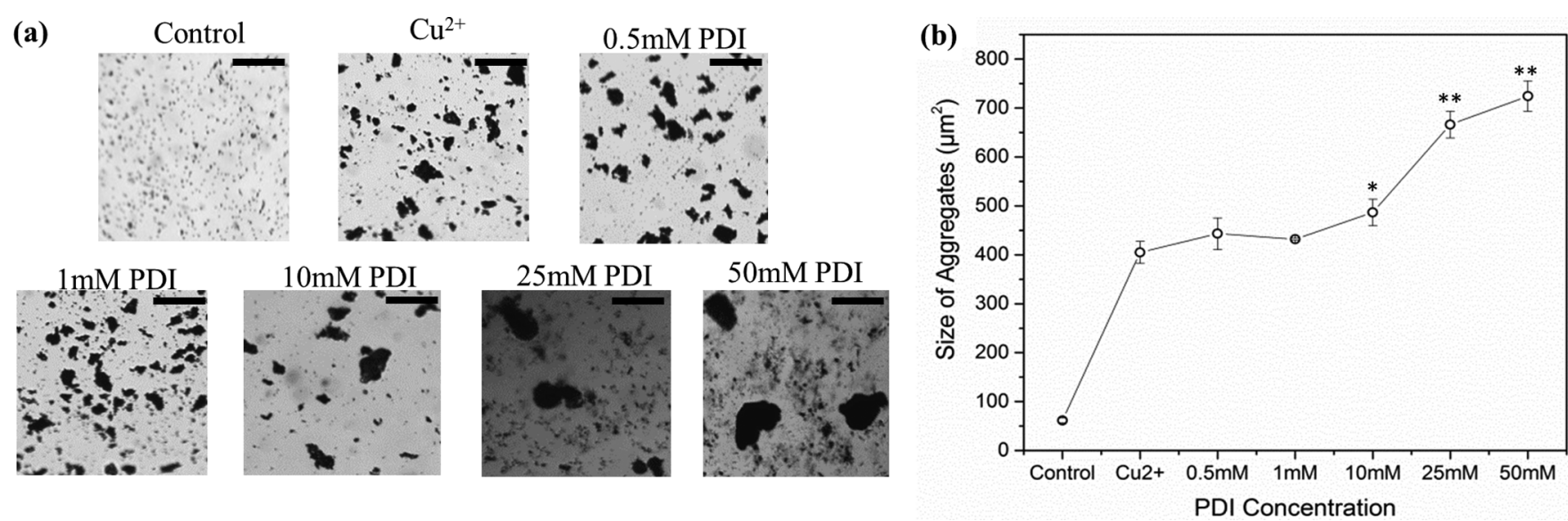

Figure 5. Effect of adding PDI at different concentrations into the MB-Cu clusters. (a) Images captured by the optical imaging unit of different MB samples. (b) Corresponding mean area of MB aggregates (calculated by Uniexplorer 6.0) vs different MB samples. Error bars indicate that the standard deviation obtained from triplicate measurements. Scale bar: $50 \mu \mathrm{m}$. $* P<0.05$, $* * P<0.01$ by one way ANOVA test compared to Cu ${ }^{2+}$ sample.

after addition of $\mathrm{Cu}$ into the MNB solution, and as millimolar concentrations of metformin were added, the $\mathrm{Cu}-\mathrm{MNB}$ clusters were disaggregated accordingly.

In our current study, we were able to test millimolar and micromolar doses of metformin due to the optimization of the assay protocol and the integration of the imaging unit. In the first experiment, we investigated the effect of higher doses $(0.5-50 \mathrm{mM})$ of metformin on $\mathrm{Cu}-\mathrm{MB}$ disaggregation (Figure 4). We found that at each concentration tested, metformin disaggregated the beads.

The control/blank sample containing only MBs suspended in MES buffer shows no MB clusters (Figure 4). Addition of $\mathrm{Cu}^{2+}$ into the control sample followed by first $\mathrm{MI}$ forms the $\mathrm{Cu}-\mathrm{MB}$ clusters. This aggregation phenomena indicates that addition of $\mathrm{Cu}^{2+}$ along with MI causes the L-cysteine-functionalized MBs to bind to $\mathrm{Cu}^{2+}$ through the formation of $\mathrm{Cu}-\mathrm{L}-\mathrm{cysteine}$ bond. Then, the addition of different concentrations of metformin into the $\mathrm{Cu}^{2+}$ sample followed by second MI causes the breakage of the clusters. As the MBs were aggregated based on the $\mathrm{Cu}-\mathrm{L}$-cysteine bond, this disaggregation phenomena indicates that metformin has caused the breakage of Cu-Lcysteine bond.
Next, we investigated the effect of the same concentrations of PDI on the Cu-MB clusters, to compare with that of metformin (Figure 5). We found that addition of PDI did not reduce the $\mathrm{Cu}-\mathrm{MB}$ clusters at lower concentrations and from $10 \mathrm{mM}$ onward, PDI increased the size of the clusters. These results demonstrate that PDI does not break the Cu-L-cysteine bond like metformin. Although they are structurally similar to metformin, malonamides like PDI are not antihyperglycemic, ${ }^{44}$ and thus, these results are consistent there being a link between the metformin's Cu-binding action and its antihyperglycemic properties.

Control Experiments. We performed control experiments in order to validate that we had measured true effects of metformin and PDI and their interaction with copper ions. In order to validate that it is not $\mathrm{MI}$ but $\mathrm{Cu}$ which forms the aggregates, a sample containing MES (copper vehicle) and functionalized MBs with a volume ratio of 11:1 (similar ratio to the $\mathrm{Cu}-\mathrm{MB}$ sample) was loaded into the disc followed by first MI which showed no clusters (Figure 6a). Then, MES buffer alone was further added to this solution followed by second MI to mimic the addition of metformin/PDI into $\mathrm{Cu}-\mathrm{MB}$ solution. Even after the second MI, no significant clusters were formed, 
(a)

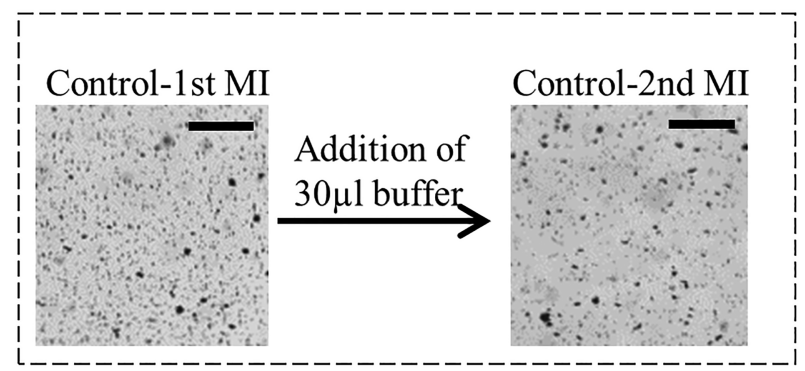

(c)

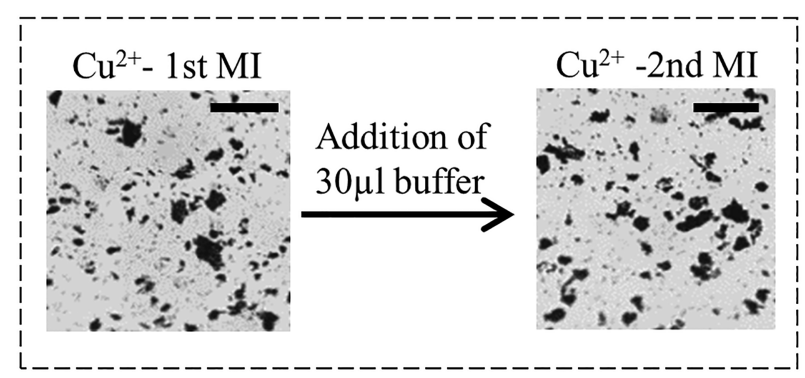

(b)

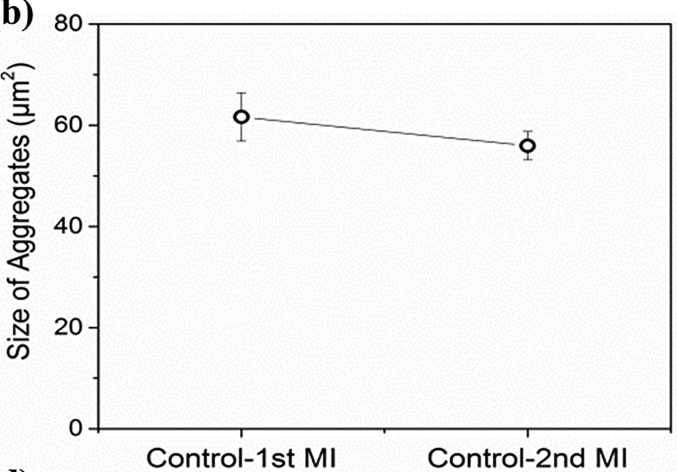

(d)

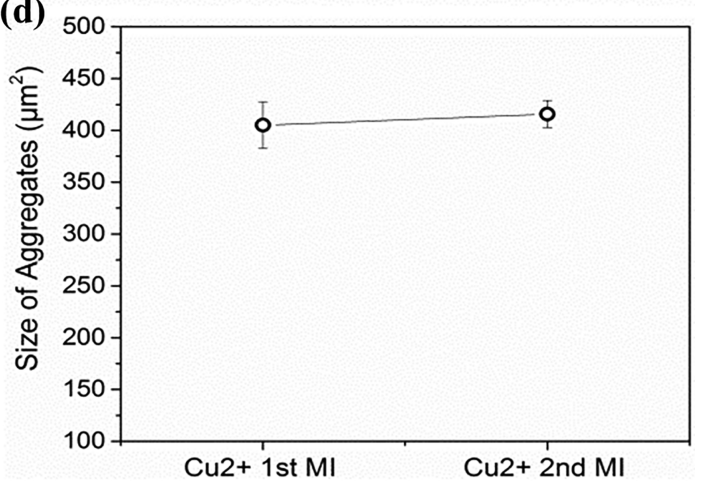

Figure 6. Control experiments to validate that the assay is measuring drug/metal interactions. (a) Images captured by the optical imaging unit showing a comparison of the MB aggregate size between the first and second MI of the control sample after adding $30 \mu \mathrm{L}$ of buffer. (b) Corresponding mean area of MB aggregates (calculated by Uniexplorer 6.0) vs the control samples. (c) Images captured by the optical imaging unit showing a comparison of the $\mathrm{Cu}-\mathrm{MB}$ aggregate size between the first and second $\mathrm{MI}$ of $\mathrm{Cu}^{2+}$ samples. (d) Corresponding mean area of $\mathrm{MB}$ aggregates (calculated by Uniexplorer 6.0) vs the $\mathrm{Cu}^{2+}$ samples. Error bars indicate the standard deviation obtained from triplicate measurements. Scale bar: $50 \mu \mathrm{m}$.

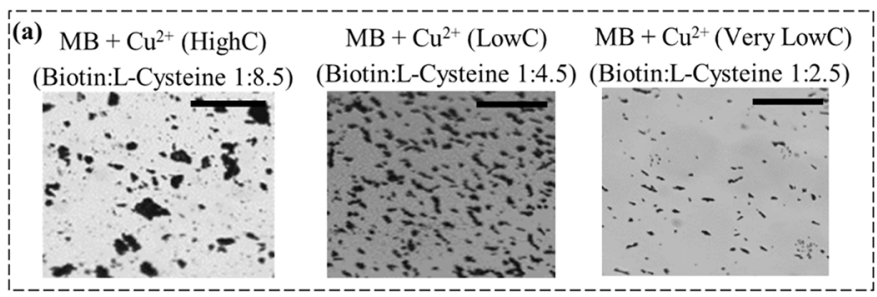

(b) Control

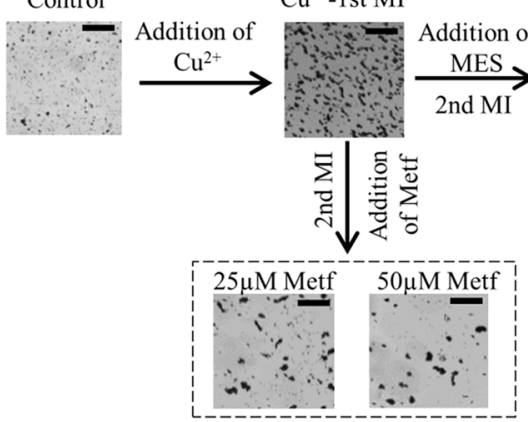

$\mathrm{MES}+\mathrm{Cu}^{2+}$

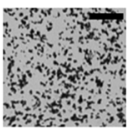

(c)

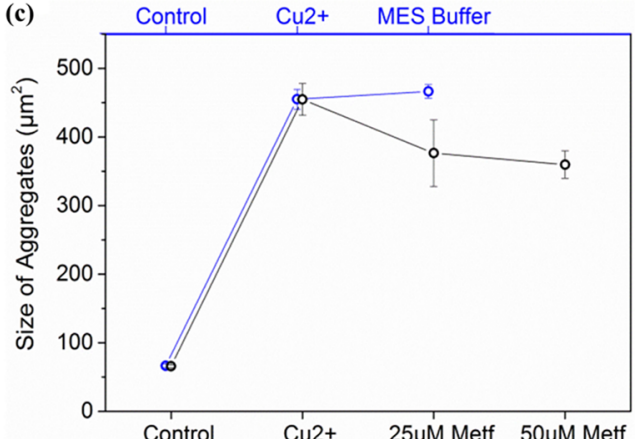

Figure 7. High sensitivity assay measures metformin/Cu interaction at physiological concentrations of the drug. (a) Images captured by the optical imaging unit showing a comparison of $\mathrm{Cu}-\mathrm{MB}$ clusters of the samples with different biotin-L-cysteine ratios. (b) Effect of low concentrations of metformin (Metf) on Cu-MB clusters using the lowC sample. Images captured by the optical imaging unit showing the effects of adding metformin at different concentrations at micromolar range into the $\mathrm{Cu}-\mathrm{MB}$ clusters. (c) Mean $\mathrm{MB}$ aggregate size (calculated by Uniexplorer 6.0) vs different MB samples. The blue line indicates the result of the control experiment which shows that addition of $\mathrm{Cu}^{2+}$ into control/blank sample creates $\mathrm{Cu}$ MB clusters followed by addition of MES showing no significant change. Error bars indicate the standard deviation obtained from triplicate measurements. Scale bar: $50 \mu \mathrm{m}$. $* P<0.05$ by one way ANOVA test compared to $\mathrm{MES}+\mathrm{Cu}^{2+}$ sample.

which shows that it is $\mathrm{Cu}$ and not $\mathrm{MI}$ which causes the larger clusters to form (Figure 6a,b).
To validate the effect of metformin/PDI on $\mathrm{Cu}-\mathrm{MB}$ clusters, we added $30 \mu \mathrm{L}$ of MES (drug vehicle) in the Cu-MB clusters 
followed by second MI which showed no significant change in cluster size (Figure 6c,d). This vehicle control strongly indicates that the effects of metformin and PDI are true drug-dependent effects. Together, these results indicate that $\mathrm{Cu}$ is required to form the large-scale aggregates and that metformin alone disaggregates these beads.

Assay Optimization to Detect Metformin/Copper Interaction at Physiological Drug Concentrations. The peak plasma concentration of metformin is in the region of 25$50 \mu \mathrm{M},{ }^{45}$ and consequently we were interested in investigating the metformin $/ \mathrm{Cu}$ interactions at these lower concentrations. In order to optimize the assay sensitivity to lower concentrations of metformin, we focused on the volume ratio of biotin and L-cysteine during the sample preparation step because the biotinylated L-cysteine is a significant component of this assay as it functions as a linker between $\mathrm{Cu}$ and streptavidin-coated MBs to enable aggregation. The initial volume ratio of biotin and L-cysteine was $1: 8.5$ by which the millimolar effects of metformin have been demonstrated above. At this volume ratio, we noted the presence of free $\mathrm{MBs}$ as well as heterogeneous $\mathrm{MB}$ clusters of smaller and larger size (Figure $7 \mathrm{a}$ ) indicating that many of $\mathrm{MBs}$ were not sufficiently functionalized with L-cysteine, due to insufficient biotinylation of L-cysteine. Probably because of this heterogeneity, we were unable to reliably detect effects of low concentrations of metformin on these MB aggregates. To improve sensitivity, we hypothesized that there might be an optimal ratio of biotin:Lcysteine, where most of the MBs would be homogeneously functionalized with biotinylated L-cysteine, leading to the formation of $\mathrm{Cu}-\mathrm{MB}$ clusters of increased homogeneity and potentially improving assay sensitivity. Optimizing $\mathrm{MB}$ functionalization with L-cysteine through maximizing the biotinylation of L-cysteine would increase the probability of MBs agglutinating more specifically through $\mathrm{Cu}$ bridges, potentially increasing the sensitivity of the assay to metformin-induced disaggregation. We varied the biotin:Lcysteine ratio in two stock solutions from 1:8.5 (highC sample) to 1:4.5 (lowC sample) and 1:2.5 (very lowC sample) by increasing the volume of biotin. The amount of L-cysteine was kept constant as reducing cysteine further below would ultimately result in a failure of aggregates to form. We compared side by side the $\mathrm{Cu}-\mathrm{MB}$ clusters formed from $\mathrm{MB}$ samples with these three different biotin and L-cysteine ratios (Figure 7a) and found indeed that the lowC ratio formed aggregates that were much more homogeneous in size and densely packed than in the highC sample.

Compared to both of these samples, the very low $\mathrm{C}$ sample, which was prepared by adding a much higher volume of biotin, resulted in $\mathrm{Cu}-\mathrm{MB}$ clusters much smaller both in size and in amount, confirming that saturation with biotin eventually blocks aggregate formation, likely because the excess amount of free biotin saturated the binding sites of streptavidin on the MBs.

We used the improved formulation of the lowC sample to study the effects of metformin at low concentrations (Figure $7 \mathrm{~b}, \mathrm{c})$. As before, the addition of $\mathrm{Cu}^{2+}$ into the $\mathrm{MB}$ solution followed by MI resulted in clusters. Further addition of $25 \mu \mathrm{M}$ or $50 \mu \mathrm{M}$ metformin followed by second MI causes the breakage of the clusters indicating that metformin does interact with $\mathrm{Cu}^{2+}$ from the $\mathrm{Cu}-\mathrm{L}-\mathrm{cysteine}$ bond, even at micromolar concentrations. To validate this experiment, MES buffer (instead of metformin) was added to $\mathrm{Cu}-\mathrm{MB}$ solution followed by MI, which showed again no significant change to the clusters.

We thus found that the formulation of the lowC sample was a crucial development to enable visualizing the effect of metformin in the micromolar range. In future work, we will continue to pursue further possibilities for optimization in order to make the assay more sensitive and robust.

\section{CONCLUSION AND FUTURE WORK}

We have developed an improved experimental setup to study molecular interactions using magnetic bead-based agglutination assay. Using this equipment along with the optimized assay, we demonstrate for the first time that metformin interacts with copper ions at physiologically significant concentrations of the drug. Furthermore, our experiments comparing the effects of metformin and PDI strongly suggest that metformin's $\mathrm{Cu}$ binding property may be linked to its therapeutic drug action. These results create a platform for future work to further investigate the effects of metformin directly in blood cells and hepatic cells at $\mu \mathrm{M}$ concentrations by adaptation of the magnetic bead-based agglutination assay we have developed. However, utilization of magnetic bead-based agglutination assay in raw biological samples (e.g., plasma or cells) is challenging as endogenous interferents can form unspecific magnetic bead clusters decreasing the specificity and sensitivity of the assay. Therefore, in our next study with raw biological samples, we will integrate the biotin-L-cysteine conjugation protocol with our previously presented ${ }^{30}$ antifouling surface architecture development through the formation of a layer of blocking proteins on the bead surface in order to prevent nonspecific aggregate formation under the influence of the biological samples. As serum albumin is well-known to minimize protein aggregation; ${ }^{46}$ likewise in a previous study, ${ }^{31}$ the surface of the MBs first would be passivated with a monolayer of human serum albumin (HSA) for preventing the formation of undesired protein corona in plasma samples. The HSA monolayer on the MB surface would be attached to L-cysteine through biotinylated biorthogonal click conjugation (e.g., $\mathrm{Cu}$ free click chemistry) forming a multilayered surface structure over MB surface for such surface structures previously demonstrated dramatic increase of assay sensitivity as well as facilitation of specific cross-linking of the affinity probes in biological fluids. $^{31,47}$ The entire assay can be integrated in the microfluidic disc with additional microfluidic structures for ondisc plasma extraction from whole blood sample. For cellular studies, a fluorescence probe can be added to the MBs with multilayered surface architecture followed by microinjecting the conjugate in hepatic (e.g., H4IIE) cells. Thus, the presence of cellular $\mathrm{Cu}$-L-cysteine is expected to form Cu-bridged $\mathrm{MB}$ clusters inside the cells while quenching the fluorescence. Adding metformin to the cell medium and its uptake into the cell will be expected to cause disintegration of the aggregates while restoring the fluorescence. Hence, the extension of this study in the future can be performed in raw biological samples by overcoming the potential challenges of biological interferences and thus adding further validation to this current study of metformin's Cu-binding action at cellular concentrations. In conclusion, the developed biosensing platform demonstrating automation, low sample-to-answer time, along with a novel application, thus paves the way for investigating further significant molecular interactions using the simple readout concept of agglutination assay in a reliable and user-friendly fashion. 


\section{ASSOCIATED CONTENT}

\section{S Supporting Information}

The Supporting Information is available free of charge on the ACS Publications website at DOI: 10.1021/acssensors.7b00384.

Description of the disc fabrication process and the detailed chemical bonding schematics of the assay (PDF)

\section{AUTHOR INFORMATION}

\section{Corresponding Author}

*E-mail: rokud@nanotech.dtu.dk. Tel: +4545256343.

ORCID $\odot$

Rokon Uddin: 0000-0002-0169-9023

Notes

The authors declare no competing financial interest.

\section{ACKNOWLEDGMENTS}

This work was financially supported by the European Research Council under the European Union's Seventh Framework Program (FP7/2007-2013) grant no. 320535-HERMES. The authors also acknowledge the support from the IDUN project (grant no. DNRF122) funded by the Danish National Research Foundation and the Velux Foundations. G. Rena acknowledges funding from MRC (MR/K012924/1).

\section{REFERENCES}

(1) Rena, G.; Pearson, E. R.; Sakamoto, K. Molecular mechanism of action of metformin: Old or new insights? Diabetologia 2013, 56 (9), $1898-1906$.

(2) Rojas, L. B. A.; Gomes, M. B. Metformin: an old but still the best treatment for type 2 diabetes. Diabetol. Metab. Syndr. 2013, 5 (1), 6.

(3) Viollet, B.; Guigas, B.; Sanz Garcia, N.; Leclerc, J.; Foretz, M.; Andreelli, F. Cellular and molecular mechanisms of metformin: an overview. Clin. Sci. 2012, 122 (6), 253-270.

(4) Rena, G.; Pearson, E. R. Hardie DGH. The mechanisms of action of metformin. Diabetologia 2017, 60, 1577.

(5) Owen, M. R.; Doran, E.; Halestrap, A. P. Evidence that metformin exerts its anti-diabetic effects through inhibition of complex 1 of the mitochondrial respiratory chain. Biochem. J. 2000, 348, 607614.

(6) El-Mir, M. Y.; Nogueira, V.; Fontaine, E.; Avéret, N.; Rigoulet, M.; Leverve, X. Dimethylbiguanide Inhibits Cell Respiration via an Indirect Effect Targeted on the Respiratory Chain Complex I. J. Biol. Chem. 2000, 275, 223-228.

(7) Foretz, M.; Hébrard, S.; Leclerc, J.; Zarrinpashneh, E.; Soty, M.; Mithieux, G.; Sakamoto, K.; Andreelli, F.; Viollet, B. Metformin inhibits hepatic gluconeogenesis in mice independently of the LKB1/ AMPK pathway via a decrease in hepatic energy state. J. Clin. Invest. 2010, 120 (7), 2355-2369.

(8) Madiraju, A. K.; Erion, D. M.; Rahimi, Y.; Zhang, X. M.; Braddock, D. T.; Albright, R. A.; Prigaro, B. J.; Wood, J. L.; Bhanot, S.; MacDonald, M. J.; Jurczak, M. J.; Camporez, J. P.; Lee, H. Y.; Cline, G. W.; Samuel, V. T.; Kibbey, R. G.; Shulman, G. I. Metformin suppresses gluconeogenesis by inhibiting mitochondrial glycerophosphate dehydrogenase. Nature 2014, 510 (7506), 542-546.

(9) Foretz, M.; Guigas, B.; Bertrand, L.; Pollak, M.; Viollet, B. Metformin: From mechanisms of action to therapies. Cell Metab. 2014, 20 (6), 953-966.

(10) Ray, R. K.; Kauffmann, G. B. Metal and Non-Metal Biguanide Complexes; New Age International Publishers: New Delhi; 1999.

(11) Ray, R. K.; Kauffmann, G. B. An EPR study of Copper (II)substituted Biguanide Complexes. Part III. Inorg. Chim. Acta 1990, 174, 237-244.
(12) Zhu, M.; Lu, L.; Yang, P.; Jin, X. Bis,(1,1-dimethylbiguanido)copper(II) octahydrate. Acta Crystallogr., Sect. E: Struct. Rep. Online 2002, 58, 217-219.

(13) Gholivand, M. B.; Mohammadi-Behzad, L. Differential pulse voltammetric determination of metformin using copper-loaded activated charcoal modified electrode. Anal. Biochem. 2013, 438 (1), $53-60$.

(14) Sharma, S. S.; Ramani, J. V.; Dalwadi, D. P.; Bhalodia, J. J.; Patel, N. K.; Patel, D. D.; Patel, R. K. New Ternary Transition Metal Complexes of $2-\backslash\{[(2$-aminophenyl)imino $]$ methyl $\}$ Phenol and Metformin: Synthesis, Characterization and Antimicrobial Activity. E-J. Chem. 2011, 8 (1), 361-367.

(15) Ray, P. Complex Compounds of Biguanides and Guanylureas With Metallic Element. Chem. Rev. 1961, 61 (4), 313-359.

(16) Logie, L.; Harthill, J.; Patel, K.; Bacon, S.; Hamilton, D. L.; Macrae, K.; McDougall, G.; Wang, H. H.; Xue, L.; Jiang, H.; Sakamoto, K.; Prescott, A. R.; Rena, G. Cellular responses to the metal-binding properties of metformin. Diabetes 2012, 61 (6), 1423-1433.

(17) Repiščák, P.; Erhardt, S.; Rena, G.; Paterson, M. J. Biomolecular mode of action of metformin in relation to its copper binding properties. Biochemistry 2014, 53 (4), 787-795.

(18) Quan, X.; Uddin, R.; Heiskanen, A.; Parmvi, M.; Nilson, K.; Donolato, M.; Hansen, M. F.; Rena, G.; Boisen, A. The copper binding properties of metformin - QCM-D, XPS and nanobead agglomeration. Chem. Commun. 2015, 51 (97), 17313-17316.

(19) Lodish, H, Berk, A, Zipursky, S. L., Matsudaira, P, Baltimore, D, Darnell, J. Electron Transport and Oxidative Phosphorylation. In Molecular Cell Biology, 4th ed.; W.H.Freeman: New York, 2000; Section 16.2 .

(20) Banci, L.; Bertini, I.; Cantini, F.; Ciofi-Baffoni, S. Cellular copper distribution: A mechanistic systems biology approach. Cell. Mol. Life Sci. 2010, 67 (15), 2563-2589.

(21) Horn, D.; Barrientos, A. Mitochondrial copper metabolism and delivery to cytochrome C oxidase. IUBMB Life 2008, 60 (7), 421-429.

(22) Yang, J.; Donolato, M.; Pinto, A.; Bosco, F. G.; Hwu, E. T.; Chen, C. H.; AlstrØm, T. S.; Lee, G. H.; Schäfer, T.; Vavassori, P.; Boisen, A.; Lin, Q.; Hansen, M. F. Blu-ray based optomagnetic aptasensor for detection of small molecules. Biosens. Bioelectron. 2016, $75,396-403$

(23) Wu, S.; Duan, N.; Wang, Z.; Wang, H. Aptamer-functionalized magnetic nanoparticle-based bioassay for the detection of ochratoxin $\mathrm{A}$ using upconversion nanoparticles as labels. Analyst 2011, 136 (11), 2306-2314.

(24) El-Boubbou, K.; Gruden, C.; Huang, X. Magnetic glyconanoparticles: A unique tool for rapid pathogen detection, decontamination, and strain differentiation. J. Am. Chem. Soc. 2007, 129 (44), 13392-13393.

(25) Horng, H. E.; Yang, S. Y.; Hong, C. Y.; Liu, C. M.; Tsai, P. S.; Yang, H. C.; Wu, C. C. Biofunctionalized magnetic nanoparticles for high-sensitivity immunomagnetic detection of human C-reactive protein. Appl. Phys. Lett. 2006, 88 (25), 252506.

(26) Tsai, H. Y.; Hsu, C. F.; Chiu, I. W.; Fuh, C. B. Detection of Creactive protein based on immunoassay using antibody-conjugated magnetic nanoparticles. Anal. Chem. 2007, 79 (21), 8416-8419.

(27) Kojima, T.; Takei, Y.; Ohtsuka, M.; Kawarasaki, Y.; Yamane, T.; Nakano, H. PCR amplification from single DNA molecules on magnetic beads in emulsion: Application for high-throughput screening of transcription factor targets. Nucleic Acids Res. 2005, 33 (17), 1-9.

(28) Wilson, K. S.; Goff, J. D.; Riffle, J. S.; Harris, L. A.; St Pierre, T. G. Polydimethylsiloxane-magnetite nanoparticle complexes and dispersions in polysiloxane carrier fluids. Polym. Adv. Technol. 2005, $16(2-3), 200-211$.

(29) Jain, T. K.; Richey, J.; Strand, M.; Leslie-Pelecky, D. L.; Flask, C. A.; Labhasetwar, V. Magnetic nanoparticles with dual functional properties: Drug delivery and magnetic resonance imaging. Biomaterials 2008, 29 (29), 4012-4021.

(30) Ranzoni, A.; Sabatte, G.; van Ijzendoorn, L. J.; Prins, M. W. J. One-step homogeneous magnetic nanoparticle immunoassay for 
biomarker detection directly in blood plasma. ACS Nano 2012, 6 (4), 3134-3141.

(31) Antunes, P.; Watterson, D.; Parmvi, M.; Burger, R.; Boisen, A.; Young, P.; Cooper, M. A.; Hansen, M. F.; Ranzoni, A.; Donolato, M. Quantification of NS1 dengue biomarker in serum via optomagnetic nanocluster detection. Sci. Rep. 2015, 5, 16145.

(32) Donolato, M.; Antunes, P.; de la Torre, T. Z. G.; Hwu, E. T.; Chen, C. H.; Burger, R.; Rizzi, G.; Bosco, F. G.; StrØmme, M.; Boisen, A.; Hansen, F. M. Quantification of rolling circle amplified DNA using magnetic nanobeads and a Blu-ray optical pick-up unit. Biosens. Bioelectron. 2015, 67, 649-655.

(33) Göransson, J. Sensitive detection of bacterial DNA by magnetic nanoparticles. Anal. Chem. 2010, 82 (22), 9138-9140.

(34) Uddin, R.; Burger, R.; Donolato, M.; Fock, J.; Creagh, M.; Hansen, M. F.; Boisen, A. Lab-on-a-disc agglutination assay for protein detection by optomagnetic readout and optical imaging using nanoand micro-sized magnetic beads. Biosens. Bioelectron. 2016, 85, 351357.

(35) Mezger, A.; Fock, J.; Antunes, P.; Østerberg, F. W.; Boisen, A.; Nilsson, M.; Hansen, M. F.; Ahlford, A.; Donolato, M. Scalable DNABased Magnetic Nanoparticle Agglutination Assay for Bacterial Detection in Patient Samples. ACS Nano 2015, 9, 7374-7382.

(36) Abajian, C.; Yatsunyk, L. A.; Ramirez, B. E.; Rosenzweig, A. C. Yeast Cox17 solution structure and copper(I) binding. J. Biol. Chem. 2004, 279 (51), 53584-53592.

(37) Boal, A. K.; Rosenzweig, A. C. Structural biology of copper trafficking. Chem. Rev. 2009, 109 (10), 4760-4779.

(38) Rubino, J. T.; Franz, K. J. Coordination chemistry of copper proteins: How nature handles a toxic cargo for essential function. $J$. Inorg. Biochem. 2012, 107 (1), 129-143.

(39) Wu, Z.; Fernandez-Lima, F. A.; Russell, D. H. Amino acid influence on copper binding to peptides: Cysteine versus arginine. $J$. Am. Soc. Mass Spectrom. 2010, 21 (4), 522-533.

(40) Zheng, H.; Chruszcz, M.; Lasota, P.; Lebioda, L.; Minor, W. Data mining of metal ion environments present in protein structures. J. Inorg. Biochem. 2008, 102 (9), 1765-1776.

(41) Cameron, A. R.; Morrison, V. L.; Levin, D.; Mohan, M.; Forteath, C.; Beall, C.; McNeilly, A. D.; Balfour, D. J.; Savinko, T.; Wong, A. K.; Viollet, B.; Sakamoto, K.; Fagerholm, S. C.; Foretz, M.; Lang, C. C.; Rena, G. Anti-Inflammatory Effects of Metformin Irrespective of Diabetes Status. Circ. Res. 2016, 119 (5), 652-665.

(42) Fredborg, M.; Andersen, K. R.; Jørgensen, E.; Droce, A.; Olesen, T.; Jensen, B. B.; Rosenvinge, F. S.; Sondergaard, T. E. Real-time optical antimicrobial susceptibility testing. J. Clin Microbiol. 2013, 51 (7), 2047-2053.

(43) Uddin, R.; Burger, R.; Donolato, M.; Fock, J.; Creagh, M.; Hansen, M. F.; Boisen, A. Integration of agglutination assay for protein detection in microfluidic disc using blu-ray optical pickup unit and optical fluid scanning. In MicroTAS 2015 - 19th International Conference on Miniaturized Systems for Chemistry and Life Sciences, 2015.

(44) Fanshawe, W. J.; Bauer, V. J.; Ullman, E. F.; Safir, S. R. Synthesis of unsymmetrically substituted malonamides. J. Org. Chem. 1964, 29 (2), 308-311.

(45) Goodman \& Gilman's The Pharmacological Basis of Therapeutics, Brunton, L. L.; Chabner, B. A.; Knollmann, B. C., Eds.; McGraw-Hill: New York, 2011.

(46) Finn, T. E.; Nunez, A. C.; Sunde, M.; Easterbrook-Smith, S. B. Serum albumin prevents protein aggregation and amyloid formation and retains chaperone-like activity in the presence of physiological ligands. J. Biol. Chem. 2012, 287 (25), 21530-21540.

(47) Sanjaya, K. C.; Ranzoni, A.; Watterson, D.; Young, P.; Cooper, M. A. Evaluation of direct versus multi-layer passivation and capture chemistries for nanoparticle-based biosensor applications. Biosens. Bioelectron. 2015, 67, 769-774. 